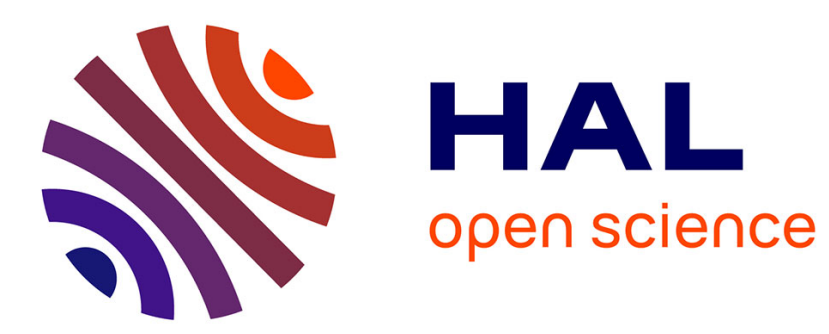

\title{
Application de la courbe diagnostique aux essais de pompage en milieu karstique
}

Jean-Christophe Maréchal, Bernard Ladouche, Benoît Dewandel, Perrine Fleury, Nathalie Dörfliger

\section{- To cite this version:}

Jean-Christophe Maréchal, Bernard Ladouche, Benoît Dewandel, Perrine Fleury, Nathalie Dörfliger. Application de la courbe diagnostique aux essais de pompage en milieu karstique. KARSTOLOGIA, 2011, 57, pp.33-36. 10.3406/karst.2011.2692 . hal-00769584

\section{HAL Id: hal-00769584 \\ https://hal.science/hal-00769584}

Submitted on 2 Jan 2013

HAL is a multi-disciplinary open access archive for the deposit and dissemination of scientific research documents, whether they are published or not. The documents may come from teaching and research institutions in France or abroad, or from public or private research centers.
L'archive ouverte pluridisciplinaire HAL, est destinée au dépôt et à la diffusion de documents scientifiques de niveau recherche, publiés ou non, émanant des établissements d'enseignement et de recherche français ou étrangers, des laboratoires publics ou privés. 


\title{
APPLICATION DE LA COURBE DIAGNOSTIQUE AUX ESSAIS DE POMPAGE EN MILIEU KARSTIQUE
}

\author{
Diagnostic plots applied to pumping tests in karst systems
}

\author{
Jean-Christophe Maréchal ${ }^{1}$, Bernard Ladouche, Benoît Dewandel, Perrine Fleury, Nathalie Dörfliger \\ ${ }^{1}$ Bureau de Recherches Géologiques et Minières (BRGM) - Service Eau, Unité NRE, 1039 rue de \\ Pinville, 34000 Montpellier, France
}

Tél: +33 (0)4 671579 65, Fax: +33 (0)4 676458 51, e-mail: jc.marechal@brgm.fr

\section{RESUME}

Les essais de pompage menés sur des puits traversant des hétérogénéités du karst telles que le réseau de drains sont difficiles à interpréter au moyen des techniques traditionnelles. Néanmoins, ce cas peut être abordé en assimilant le conduit karstique horizontal à une fracture verticale de perméabilité finie. Dans ce cas, plusieurs types d'écoulements correspondant aux contributions respectives des soussystèmes du karst (matrice fracturée de la zone noyée, petits conduits, réseau de drainage principal) peuvent être identifiés sur la courbe diagnostique de la dérivée du rabattement. Cette méthode est illustrée sur deux cas réels provenant d'aquifères karstiques méditerranéens du sud de la France. Un essai de pompage réalisé sur un puits traversant le réseau de drainage karstique principal du système des Cent-Fonts montre (1) une première contribution du stockage capacitif au sein du conduit karstique suivi (2) d'écoulements monodimensionnels au sein de la matrice fracturée. Un essai de pompage sur un puits traversant à grande profondeur un petit conduit karstique du système des Corbières montre l'existence (1) d'un écoulement bilinéaire au sein du conduit et de la matrice fracturée au début, suivi (2) d'écoulements radiaux au sein de la matrice fracturée et enfin (3) de la contribution d'une cavité karstique majeure. L'utilisation de la courbe diagnostique établie à l'aide de la dérivée des rabattements permet d'identifier les différents régimes d'écoulements durant un essai de pompage, correspondant aux réponses successives des sous-systèmes de l'aquifère karstique. Ceci contribue à mieux comprendre la structure de l'aquifère karstique ainsi que les échanges de flux entre sous-systèmes.

\begin{abstract}
Pumping tests conducted on wells intersecting karst heterogeneities such as the conduit network are difficult to interpret. Nevertheless, this case can be solved by assimilating the horizontal karst conduit to a finite-conductivity vertical fracture. In this case, several flow patterns corresponding to the respective contributions of karst subsystems (fractured matrix, small conduits and main karst drainage network) can be identified on the diagnostic plot of drawdown derivative. This is illustrated on two examples from Mediterranean karst systems in southern France. A pumping test on a well intersecting the main karst drainage network of the Cent-Fonts karst system shows (i) a preliminary contribution of the karst conduit storage capacity followed by (ii) linear flows into the fractured matrix. A pumping test on a well intersecting a small karst conduit of the Corbières karst system shows the existence of (i) bi-linear flow within both the karst conduit an the fractured matrix at early times, followed by (ii) radial flows within the fractured matrix and (iii) finally the contribution of a major karst cavity. The use of diagnostic plots allows identifying the various flow regimes during pumping tests,
\end{abstract}


corresponding to the response of the individual karst aquifer subsystems. This is helpful for improving the understanding of the structure of the karst aquifer and flow exchanges between subsystems.

\section{MOTS CLEFS}

Essai de pompage, système karstique, hydrogéologie, rabattement, perméabilité

\section{KEYWORDS}

Pumping test, karst system, hydrogeology, drawdown, permeability

\section{INTRODUCTION}

Suite à la complexité et la dualité des écoulements dans les aquifères karstiques, l'interprétation des essais de pompage dans ces milieux constitue une tâche difficile pour les hydrogéologues (Kresic 2007). Cette constatation est particulièrement vraie dans le cas d'un puits interceptant une hétérogénéité karstique telle qu'un réseau de conduits par exemple. Pourtant, les essais de pompage peuvent apporter des informations intéressantes quant à la présence ou la taille des hétérogénéités karstiques.

La courbe de dérivée des rabattements, appelée également courbe diagnostique (« diagnostic plot »), a été largement appliquée en prospection pétrolière à la caractérisation des réservoirs fracturés. Cette méthode est maintenant de plus en plus utilisée par les hydrogéologues afin d'identifier les modèles d'écoulements appropriés aux aquifères fracturés en pompage (Maréchal et al. 2004; Renard et al. 2009). Ce type d'analyse peut également être appliqué aux aquifères karstiques (Maréchal et al. 2008). Dans cet article, la réponse classique d'un essai de pompage dans une importante fracture (ou, par analogie un conduit karstique) est décrite sur un graphique log-log des dérivées des rabattements. Cela permet d'identifier les phases successives des régimes d'écoulement correspondant à la contribution de différents compartiments du karst (matrice fracturée dans la zone saturée, conduit karstique, réseau principal de drains karstiques) au puits de pompage. L'interprétation d'une telle courbe de dérivées est illustrée sur deux exemples dans des systèmes karstiques méditerranéens du sud de la France.

\section{METHODE}

Au sein des systèmes karstiques, le graphe log-log du rabattement au puits de pompage et dans les piézomètres d'observation ainsi que sa dérivée (courbe diagnostique) peut aider à identifier des régimes d'écoulements souterrains différents du cas classique radial et homogène. Le logarithme du rabattement $s$ est représenté en fonction du logarithme du temps $t$, avec $\log (\mathrm{d} s / \mathrm{d} \ln t)$, le logarithme de la dérivée du rabattement en fonction du logarithme du temps. Plusieurs auteurs (Ehlig-Economides 1988) ont observé que la dérivée du rabattement prend la forme de lignes droites pour les temps longs, avec des pentes variant en fonction de la dimension d'écoulement du milieu et des conditions aux limites. Classiquement, le graphe log-log de la dérivée peut être divisé en plusieurs phases avec : (1) les données aux temps courts pour identifier l'effet capacitif dans le puits et/ou le conduit karstique (2) les données intermédiaires pour identifier le type de modèle d'aquifère qui devrait être utilisé (par exemple double porosité, aquifère libre, fracture unique); et (3) les données aux temps longs pour mettre en évidence les éventuelles conditions aux limites (limites imperméables, grandes cavités karstiques). 
D'un point de vue opérationnel, l'implantation d'un puits de pompage sur le réseau de drains (Figure 1) d'un système karstique constitue une cible intéressante pour la future exploitation d'un aquifère karstique. Cette position du puits de pompage permet de drainer le conduit karstique fortement perméable qui va ensuite interagir avec la matrice avoisinante. Le conduit karstique constitue alors une extension très utile du puits de pompage. Deux cas de figures sont distingués : (a) le cas d'un puits de pompage interceptant le réseau principal de drains karstiques connecté à l'exutoire du système ( $\mathrm{Pa}$, Figure 1) et (b) le cas d'un puits de pompage interceptant un petit conduit karstique $(\mathrm{Pb}$, Figure 1).

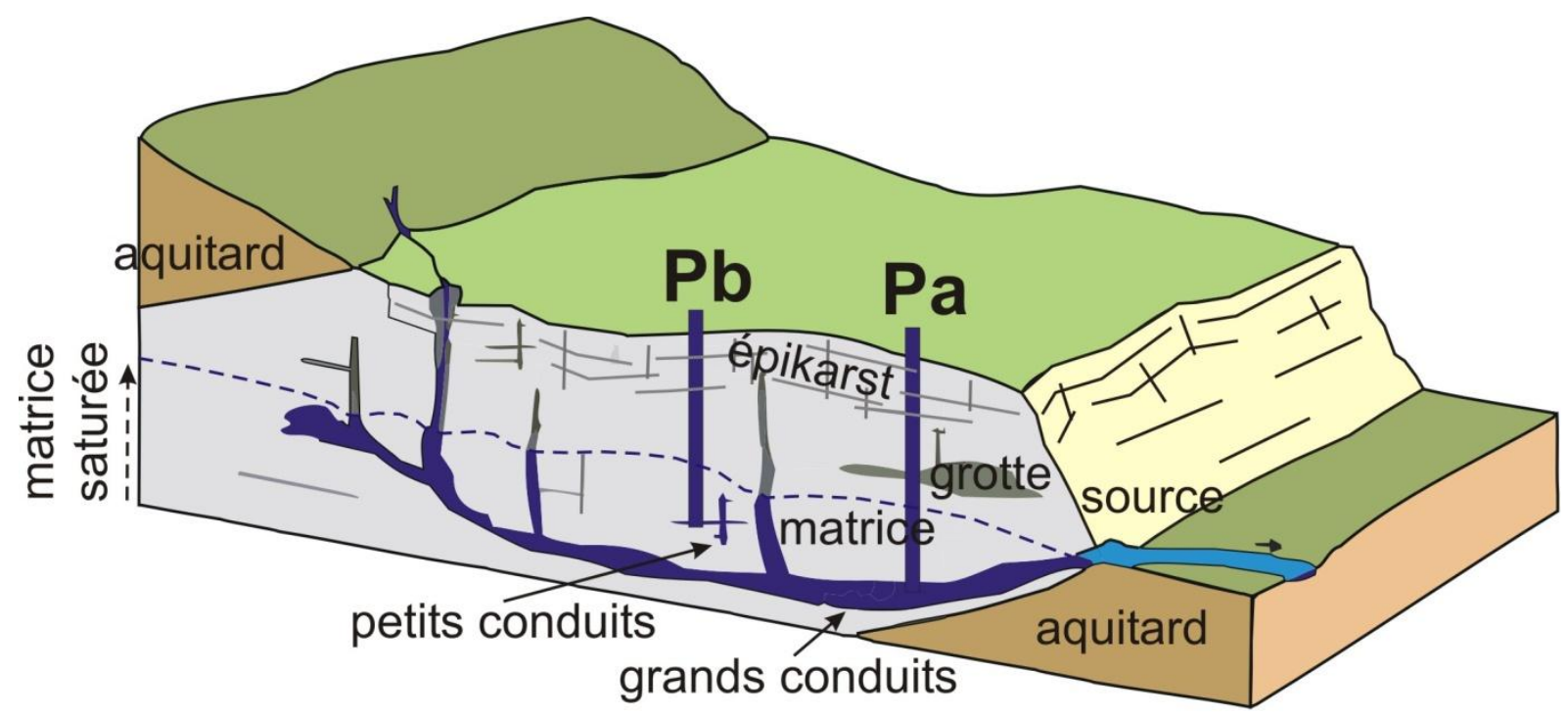

Figure 1 : aquifère karstique avec des puits de pompages $(P)$ localisés au sein du réseau de conduits karstiques (modifié d'après Goldscheider \& Drew 2007) - Pa : puits de pompage interceptant le réseau principal de drains karstiques, $\mathrm{Pb}$ : puits interceptant un petit conduit karstique

Ces deux cas peuvent être comparés au cas d'un forage stimulé par fracturation hydraulique tel que décrit dans la littérature des pétroliers (Gringarten \& Ramey 1974; Ramey \& Gringarten 1976). Considérons un puits au sein d'une fracture verticale produisant à un débit constant au sein d'un aquifère horizontal, homogène, infini et isotrope. La figure 2 représente le comportement général d'un puits avec un conduit horizontal qui peut être assimilé à une fracture verticale de perméabilité donnée. La géométrie de l'écoulement dans de telles conditions va changer avec le temps et inclure différentes périodes d'écoulements (Cinco-Ley \& Samaniego-V 1981). Au début du pompage (temps courts, Figure 2a), l'écoulement linéaire dans le conduit (effet capacitif du puits ou du conduit) est caractérisé par une droite de pente $v=1$ sur la courbe diagnostique. Après une période d'écoulement de transition, le système montre (ou pas) une période d'écoulements bilinéaires (Figure 2b), indiquée par une droite de pente $v=1 / 4$ qui dure tant que les limites du conduit n'affectent pas le régime d'écoulement. Avec le temps croissant, l'écoulement dans la matrice devient monodimensionnel (horizontal et perpendiculaire au conduit) comme illustré à la Figure 2c. Sur une courbe diagnostique, la pente de la dérivée est $v=0.5$. Si le pompage continue, le régime d'écoulement peut passer d'un régime de type parallèle à un régime pseudo-radial (Figure 2d). Durant cette période, l'eau provient de plus loin et l'effet local du conduit de longueur finie tend à disparaître. La courbe de rabattement tend vers la droite de Jacob avec une pente $v=0$ des dérivées aux temps longs, mais ceci ne se produit que si la durée de l'essai de pompage est très longue. Cette géométrie des écoulements en quatre phases rend impossible l'interprétation d'une telle courbe de rabattements au moyen des techniques 
classiques. Les courbes de rabattements et de dérivées résultantes sont représentées à la Figure 2e. Chaque cas d'étude ne présente pas systématiquement les quatre phases d'écoulements décrites à la Figure 2. Selon les caractéristiques géométriques du conduit karstique (longueur, diamètre), les paramètres hydrodynamiques du milieu (contraste de perméabilité entre matrice et conduit karstique), la présence de conditions aux limites spécifiques ou la durée de l'essai de pompage, certaines phases d'écoulement n'apparaissent pas sur la courbe diagnostique. Ceci est illustré ci-dessous sur les systèmes karstiques des Cent-Fonts et des Corbières.

\section{(a) Ecoulement linéaire (conduit)}

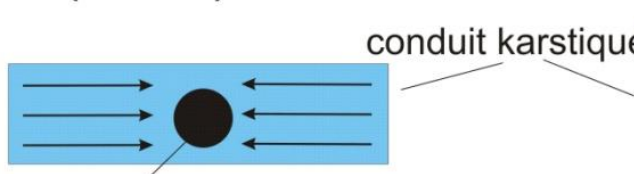

puits de pompage

(c)

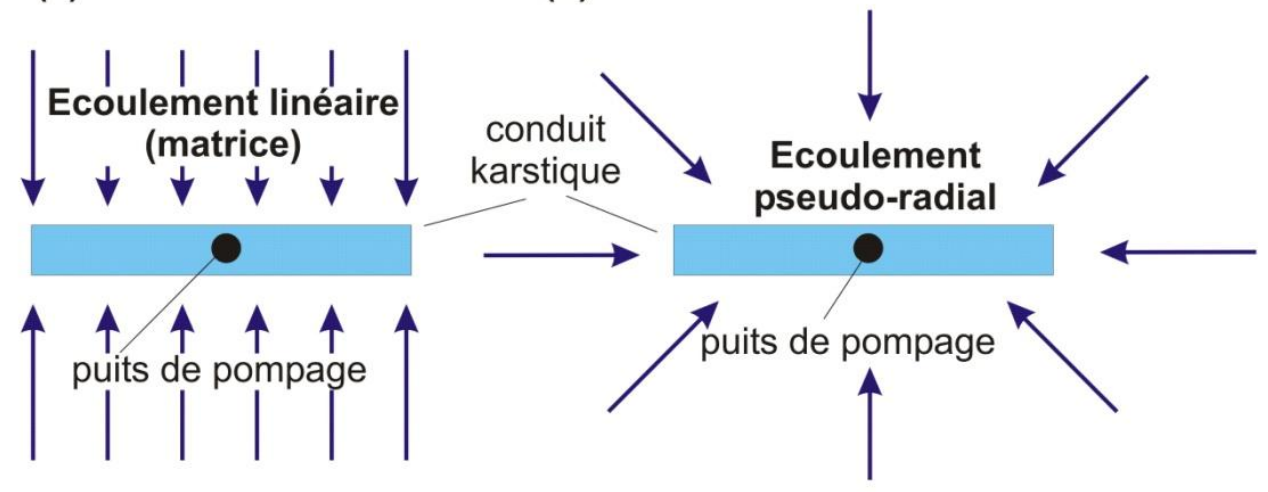

(b)
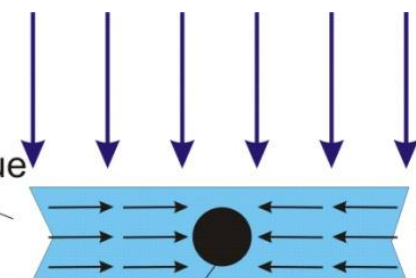

\section{Ecoulement} bilinéaire

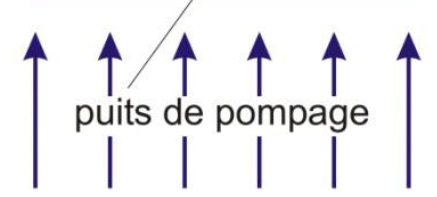

(d)

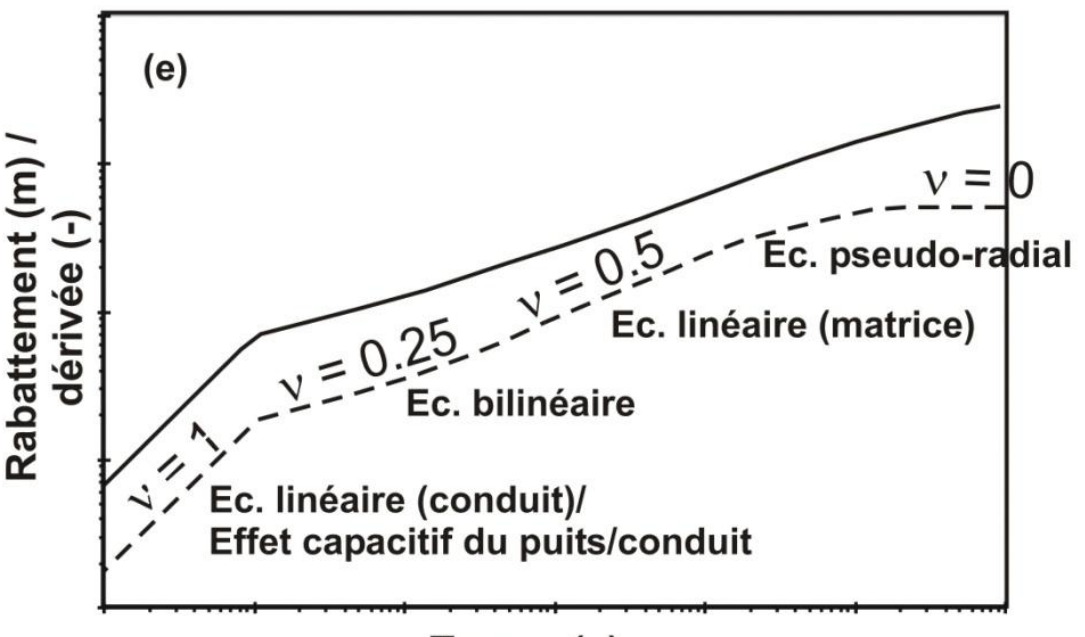

Temps (s)

Figure 2 : réponse d'un puits interceptant un conduit karstique se comportant comme une fracture vertical de longueur finie et de perméabilité finie: (a) Ecoulement linéaire dans le conduit ou effet capacitif du puits/conduit aux temps courts; (b) Ecoulements bilinéaires; (c) Ecoulement linéaire dans la matrice; (d) Ecoulement pseudo-radial dans la matrice au cours des 


\section{temps longs, modifié d'après Cinco-Ley \& Samaniego-V, 1981; (e) Courbe diagnostique résultante}

\section{RESULTATS}

\section{III.1 Système karstique des Cent-Fonts}

Un essai de pompage de longue durée (un mois à partir de fin juillet 2005) a été mené sur le conduit principal du système karstique des Cent-Fonts (Maréchal et al. 2008) durant une période d'étiage fort. Le débit à la source était de $255 \mathrm{l} / \mathrm{s}$ en début d'essai, le plus faible mesuré depuis le début du suivi hydrologique en 1997. Un puits d'une profondeur de 128 mètres a été implanté sur base d'explorations menées par des plongeurs afin d'intercepter le conduit karstique 100 mètres en amont de l'exutoire du système situé le long du fleuve Hérault sur le territoire de la commune de Causse-dela-Selle. Excepté un arrêt de quelques heures après 8 jours (soit environ 12000 minutes sur les diagrammes de la Figure 3), le puits a été pompé à un débit constant de $400 \mathrm{l} / \mathrm{s}$ provoquant le tarissement total de la source durant le pompage et ce, en l'absence de précipitation. Durant les premières 24 heures (environ 1500 minutes), l'effet capacitif au sein du conduit karstique domine (pente $v=1$, Figure 3a). Ensuite, les rabattements tendent vers un écoulement linéaire avec une pente décroissant à $v=0.7$. Aucune période d'écoulement bilinéaire n'a été observée. Dans les milieux fracturés, Cinco-Ley \& Samianego-V (1981) considèrent que ce comportement n'est pas présent quand la fracture possède un fort effet capacitif et une forte perméabilité. Dans ce cas précis, c'est la raison la plus probable d'un tel comportement hydrodynamique étant donné le large diamètre $(3,5$ mètres) du conduit karstique au voisinage du puits de pompage. Les variations de conductivité électrique de l'eau pompée (Figure 3a) ne sont pas corrélées avec le comportement hydrodynamique du système.

Les solutions analytiques pour un puits de pompage interceptant une fracture verticale de perméabilité infinie peuvent être transposées à un puits interceptant un conduit karstique très perméable. Par analogie, le conduit karstique peut être assimilé à une fracture et la matrice fracturée à un milieu poreux homogène. De telles solutions ont été développées par les ingénieurs pétroliers pour leur application à des puits fracturés artificiellement (Kruseman \& Ridder 1990). Elles prennent en compte un puits complet fracturé verticalement au sein d'une formation horizontale homogène d'épaisseur constante et de porosité $\left(S_{m}\right)$ et transmissivité $\left(T_{m}\right)$.

Dans le cas où l'effet capacitif du conduit karstique est négligeable, la fracture peut être identifiée à une surface plane d'épaisseur nulle et de perméabilité infinie, avec un rabattement nul au sein de celle-ci. Par ailleurs, si le puits ou le conduit karstique est suffisamment grand pour générer un effet capacitif significatif, une solution modifiée peut être appliquée (Gringarten \& Ramey 1974; Ramey \& Gringarten 1976). Ce volume capacitif inclut tous les volumes de forte perméabilité qui communiquent avec le puits. Il est représenté par $C_{v f}$, défini comme le rapport entre la variation de volume d'eau dans le puits et le drain karstique, et le rabattement correspondant. Ce paramètre hydrodynamique correspond à la surface libre du réseau de conduits karstiques se désaturant en cours de pompage (cheminées verticales et conduits variablement saturés). Bien que citées dans la littérature hydrogéologique, des cas pratiques de ces solutions analytiques sont rares (Kruseman \& Ridder 1990). 


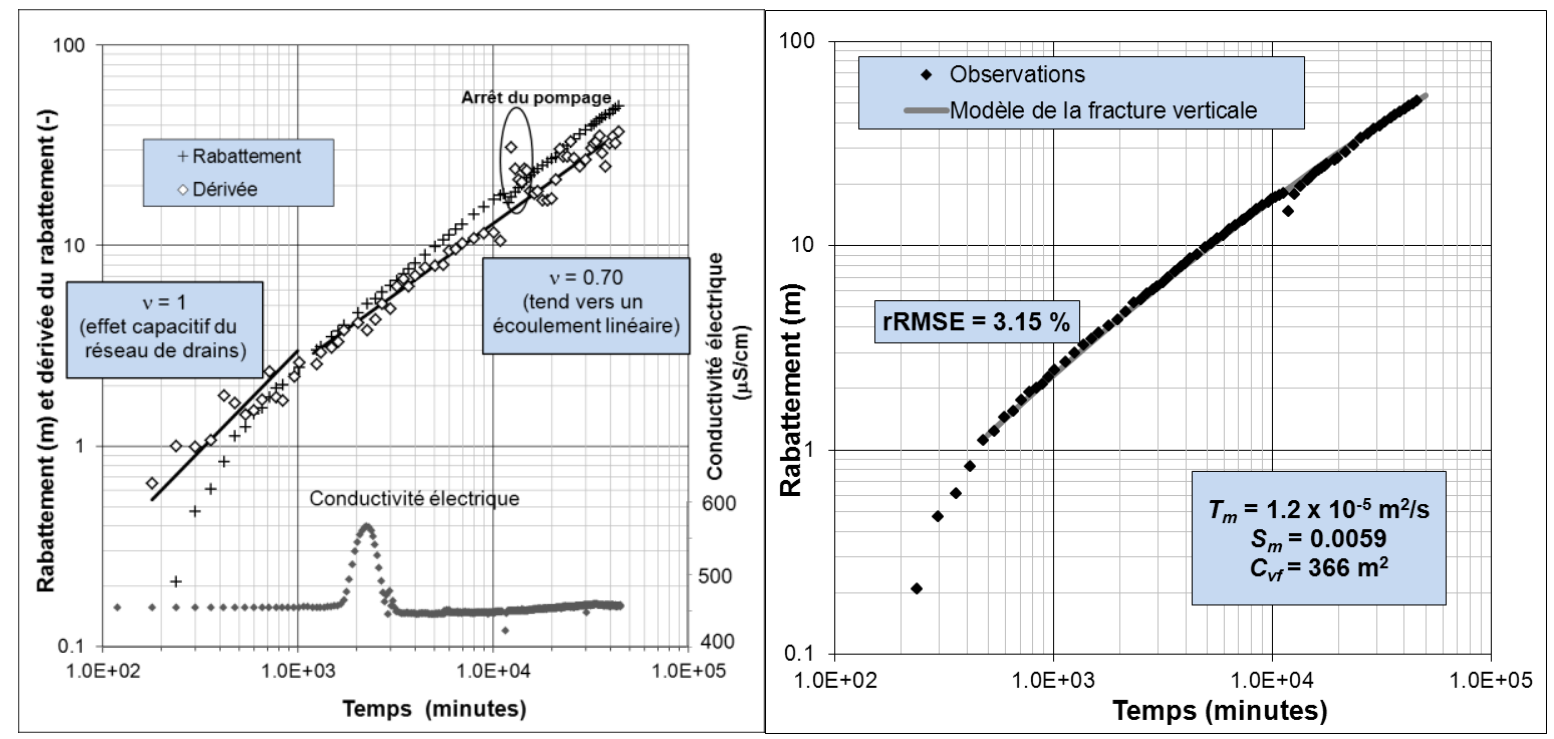

Figure 3: (a) Courbe diagnostique du pompage Cent-Fonts; (b) Evolution temporelle du rabattement mesuré et de sa modélisation par la solution de la fracture verticale (Ramey \& Gringarten, 1976), modifié d'après Maréchal et al., 2008

Le modèle de fracture verticale avec effet capacitif s'ajuste bien aux observations après 10 heures de pompage avec une erreur relative (relative root mean squared error - rRMSE) de 3.15\% (Figure 3b). En assumant l'hypothèse d'un conduit karstique de 5000 mètres de longueur, les paramètres hydrodynamiques obtenus sont donnés à la Figure 3b. Durant ce test, aucun écoulement pseudo-radial n'a été observé. Cela est probablement dû à la longueur du réseau de conduits karstiques.

\section{III.2 Système karstique des Corbières}

Le puits Robol a été foré au travers des calcaires des Corbières sur le territoire de la commune de Salses-le-Château près de Perpignan dans les Pyrénées Orientales (sud de la France). Avec une profondeur totale de 500 mètres, ce forage a traversé un conduit karstique d'un diamètre approximatif de $10 \mathrm{~cm}$ à 420 mètres de profondeur. En juin 2009, durant une période d'étiage du système karstique et en l'absence de précipitation, un essai de pompage d'une durée de 5 jours a été réalisé à un débit constant de $45 \mathrm{~m}^{3} / \mathrm{h}$ pendant les 56 premières heures puis $51 \mathrm{~m}^{3} / \mathrm{h}$ jusqu'à la fin de l'essai. La courbe diagnostique de l'essai de pompage de longue durée montre l'existence de trois phases d'écoulements (Figure 4):

- Durant les 20 premières minutes, le régime d'écoulement est caractérisé par une pente $v=$ 0.2 - 0.3 correspondant à un écoulement bilinéaire (écoulements linéaires dans le conduit et dans la matrice fracturée);

- $\quad$ Entre 20 et 100 minutes, la dérivée du rabattement est constante $(v=0)$ correspondant à un régime d'écoulement pseudo-radial au sein de la matrice. La transmissivité de la matrice a été estimée à $2.5 \times 10^{-4} \mathrm{~m}^{2} / \mathrm{s}$; 
- Après 100 minutes, la dérivée des rabattements décroit fortement à cause d'un rabattement stabilisé lié à une condition aux limites de type potentiel imposé probablement liée à la contribution d'un important volume karstique.

Aucun régime d'écoulement linéaire n'a été observé au début du test du fait du faible diamètre du conduit karstique traversé et de son faible effet capacitif associé. La transition rapide à un écoulement de type pseudo-radial est liée à la longueur limitée du conduit karstique comparée, par exemple, au conduit karstique des Cent-Fonts. Dès lors, aucun écoulement linéaire n'est observé dans la matrice fracturée.

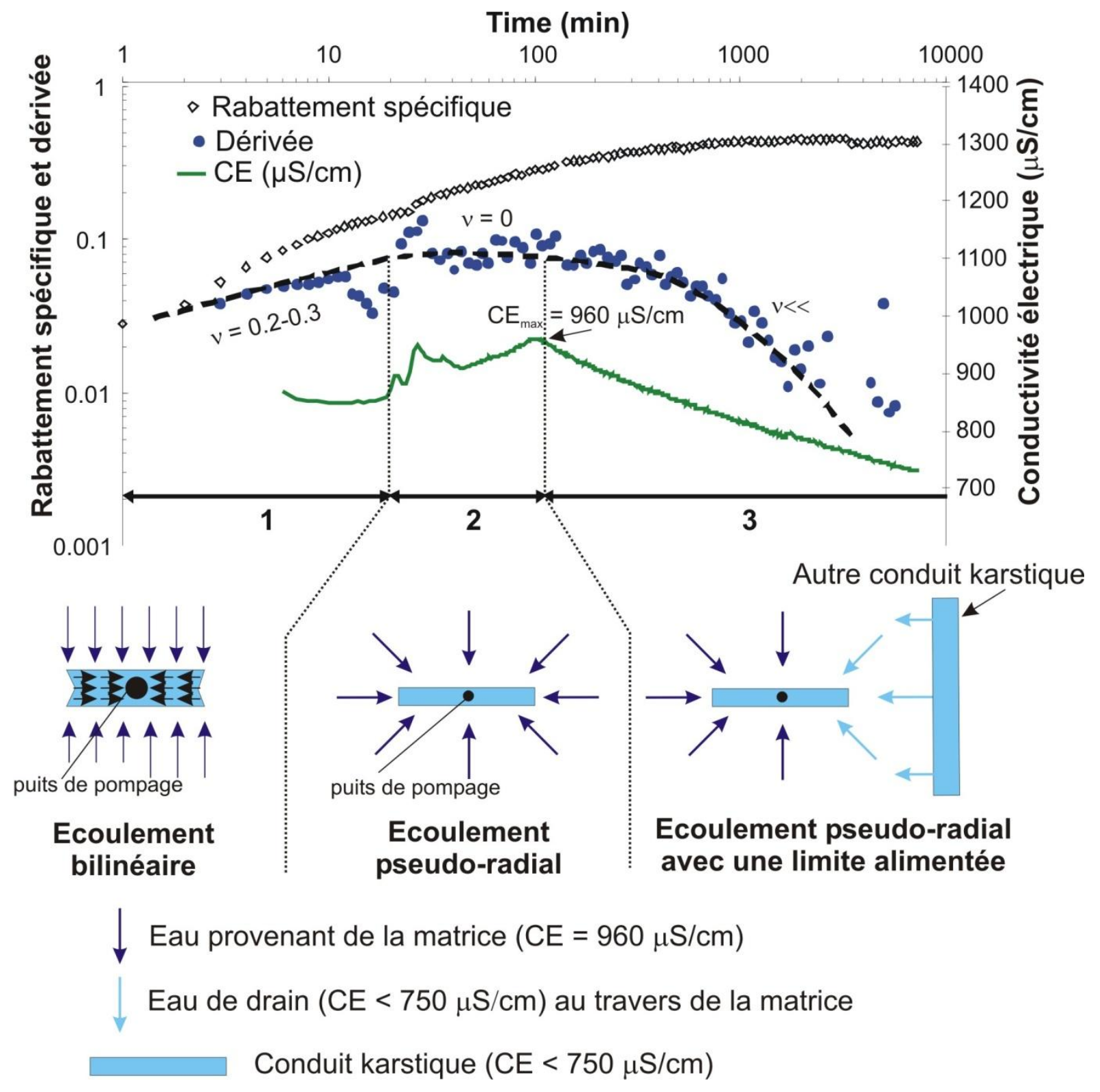

Figure 4 : essai de pompage au puits Robol, interceptant un petit conduit karstique profond

Ces changements dans les régimes d'écoulements induisent des fluctuations de minéralisation de l'eau pompée qui peut être suivie par la conductivité électrique (Fig. 4). Faisons l'hypothèse que l'eau pompée résulte du mélange de deux pôles contributeurs : une eau moins minéralisée (faible conductivité électrique) provenant du conduit karstique et une eau plus minéralisée (conductivité électrique élevée) provenant de la matrice. Durant la première phase (Phase 1), la conductivité 
électrique est médiane du fait que l'eau est un mélange d'eau de conduit et d'eau de matrice. Durant la seconde phase (Phase 2), la conductivité électrique augmente au gré de l'augmentation de contribution de la matrice: la valeur maximale atteint $C E_{\max }=960 \mu \mathrm{S} / \mathrm{cm}$. Une fois que l'effet d'un autre volume karstique est observé sur la dérivée du rabattement (Phase 3), la conductivité électrique de l'eau décroît vers celle de l'eau du conduit karstique $(C E \leq 750 \mu \mathrm{S} / \mathrm{cm})$. La diminution de conductivité électrique suggère l'existence d'une relation entre les volumes karstiques. Ceci pourrait être lié à un réseau de fractures entre deux conduits karstiques séparés ou une constriction séparant un unique conduit karstique en deux parties. De façon intéressante, l'augmentation forte de conductivité électrique de l'eau après 20 à 25 minutes induite par l'augmentation de débit de pompage était liée à l'intrusion d'eau de matrice; le petit conduit karstique pompé ne pouvant fournir l'eau nécessaire, fournie en conséquence par la matrice fracturée.

\section{CONCLUSION}

Les méthodes classiques pour l'interprétation des essais de pompage ne peuvent pas être appliquées aux puits traversant des hétérogénéités karstiques. Néanmoins, l'utilisation des courbes de diagnostique permet d'identifier des régimes d'écoulements variant au cours de l'essai et correspondant à la contribution des différents sous-systèmes de l'aquifère karstique (matrice fracturée, petits conduits, et principaux conduits karstiques). La succession de différents régimes d'écoulements apporte de l'information sur la complexité des écoulements au sein du système karstique en pompage. La courbe diagnostique permet d'identifier la solution analytique adaptée pour déterminer les paramètres hydrodynamiques de l'aquifère karstique. Les variations de conductivité électrique en cours de pompage permettent dans certains cas de valider le diagnostic effectué sur les rabattements.

\section{REMERCIEMENTS}

Ce projet de recherche a été financé par le BRGM dans le cadre des projets Corbières Phase 3 et EAUR15-R2EAU. Les Conseils Généraux de l'Aude et des Pyrénées Orientales, le Conseil Régional du Languedoc-Roussillon et l'agence de l'eau Rhône-Méditerranée et Corse (AERM\&C) ont également financé ce projet de recherche.

\section{REFERENCES}

Cinco-Ley H. \& Samaniego-V F. 1981 - Transient pressure analysis for fractured wells. Journal of Petroleum Technology, 33(9), p. 1749-1766.

Ehlig-Economides C. 1988 - Use of the pressure derivative for diagnosing pressure-transient behavior. Journal of Petroleum Technology, p. 1280-1282.

Goldscheider N. \& Drew D.P. 2007 - Methods in Karst hydrogeology. Taylor \& Francis Group.

Gringarten A.C. et Ramey H.J. 1974. Unsteady-state pressure distributions created by a well with a single infinite-conductivity vertical fracture. Society of Petroleum Engineers Journal, August, p. 347360.

Kresic N. 2007 - Hydraulic methods, in: Methods in karst hydrogeology / edited by Nico Goldscheider \& David Drew. Taylor \& Francis Group.

Kruseman G.P. et Ridder N.A. 1990 - Analysis and evaluation of pumping test data. ILRI Publication., $377 \mathrm{p}$. 
Maréchal J.C., Dewandel B. et Subrahmanyam K. 2004. Use of hydraulic tests at different scales to characterize fracture network properties in the weathered-fractured layer of a hard rock aquifer. Water Resources Research, 40(11).

Maréchal J.C., Ladouche B., Dörfliger N. et Lachassagne P. 2008 - Interpretation of pumping tests in a mixed flow karst system. Water Resources Research, 44(5).

Ramey H.J. et Gringarten A.C. 1976 - Effect of high-volume vertical fractures on geothermal steam well behavior. U.S. Government Printing Office, p. 1759-1762.

Renard P., Glenz D. \& Mejias M. 2009 - Understanding diagnostic plots for well-test interpretation. Hydrogeology Journal, 17(3), p. 589-600.

\section{Légende des photos}

Photo1: plateforme de pompage sur le puits F3 aux Cent-Fonts

Photo2 : exhaure des eaux de pompage dans le fleuve Hérault durant l'essai aux Cent-Fonts 